\title{
REMEDIACION DE RESIDUOS SÓLIDOS CONTAMINADOS CON Cr(VI) POR UN HONGO FILAMENTOSO
}

\author{
(Remediation of waste contaminated with $\mathrm{Cr}$ (VI) by a filamentous fungus)
}

${ }^{1}$ Antonela Márquez A.; ${ }^{1}$ Ignacio Busnelli G.; ${ }^{2}$ Gladys Duca D. \& ${ }^{1 *}$ Cristina Rubio M.

1Instituto de Biotecnología. Facultad de Bioquímica, Química y Farmacia.

${ }^{2}$ Facultad de Medicina. Universidad Nacional de Tucumán.

Ayacucho 471 (4000). Tucumán. Argentina. Tel: 54-03814107209.

*Autor para correspondencia: mcrubio@fbqf.unt.edu.ar

RECIBIDO:14 de Diciembre de 2014

APROBADO:10 de Marzo de 2015

LOS AUTORES DECLARAN NO TENER CONFLICTO DE INTERESES

Palabras claves: Aspergillus niger, Biorremediación, Cr(VI), residuos sólidos

Key words: Aspergillus niger, Bioremediation, Chromium, solid waste

\section{RESUMEN}

La biotecnología ambiental recurre a organismos capaces de reducir los niveles de metales pesados, entre ellos el $\mathrm{Cr}(\mathrm{VI})$, contenido en residuos y efluentes agroindustriales. El objetivo del trabajo fue estudiar la biorremediación de un residuo como pulpa de limón contaminada con $\mathrm{Cr}(\mathrm{VI})$ y el efecto del metal sobre el crecimiento fúngico. Se utilizaron tres hongos filamentosos como Aspergillus niger; Penicillium expansum y P. islandicum para remediar pulpa de limón (residuo) contaminada con $\mathrm{Cr}(\mathrm{Vl})(50 \mathrm{mg} / \mathrm{L})$ que se realizó en las siguientes condiciones de cultivo: la pulpa se suplementó con urea, 0,006; $\left(\mathrm{NH}_{4}\right)_{2} \mathrm{SO}_{4}$, 0,$012 ; \mathrm{KH}_{2} \mathrm{PO}_{4}, 0,003$ y KCl, $0,001 \mathrm{~g} / \mathrm{g} ; 10^{5}$ conidios/ $\mathrm{g}$, a pH $2,5,30^{\circ} \mathrm{C}$ y $96 \mathrm{~h}$ de incubación. Se estudió el efecto tóxico de diferentes concentraciones $(5 ; 10 ; 20$ y $50 \mathrm{mg} / \mathrm{L}$ ) del metal sobre el desarrollo del hongo de mayor eficiencia de remediación (Ef.\%). Aspergillus niger; obtuvo mayor EF. de remediación (97\%) respecto a Penicillium expansum (95\%) y P. islandicum (94\%), del residuo contaminado con $50 \mathrm{mg} / \mathrm{L} \mathrm{de}$ $\mathrm{Cr}(\mathrm{VI})$. Se determinó que la presencia de $\mathrm{Cr}(\mathrm{VI})$ y no su concentración estimuló la maduración temprana (48 h) de los conidios (blancos a negros) de A. niger, sin que se observe alteraciones en el micelio con respecto al control (72h), desarrollado en la pulpa sin el metal. En conclusión, A. niger fue más resistente y presentó altas Ef. de remediación de $\mathrm{Cr}(\mathrm{VI})$ de residuos sólidos, este proceso es una alternativa a las tecnologías físico-químicas, debido que los microorganismos pueden remover selectivamente diferentes iones de zonas contaminadas.

\section{ABSTRACT}

Environmental biotechnology uses organisms capable of reducing levels of heavy metals, including the $\mathrm{Cr}(\mathrm{VI})$, contained in waste and agro-industrial effluents. The objective of this work was to study bioremediation of waste contaminated with $\mathrm{Cr}(\mathrm{VI})$ lemon pulp and the effect of the metal on the fungal growth. We used three filamentous fungi such as Aspergillus niger; Penicillium expansum and $P$. islandicum to remedy pulp from lemon (residue) contaminated with $\mathrm{Cr}(\mathrm{VI})(50 \mathrm{mg} / \mathrm{L})$ that was conducted in the following conditions of cultivation: the pulp is supplemented with urea, 0.006; $(\mathrm{NH} 4)_{2} \mathrm{SO}_{4}$, $0.012 ; \mathrm{KH}_{2} \mathrm{PO}_{4}, 0.003$ and $\mathrm{KCl}, 0.001 \mathrm{~g} / \mathrm{g} ; 10^{5}$ conidia/g, at pH $2.5,30^{\circ} \mathrm{C}$ and $96 \mathrm{~h}$ of incubation. We studied the toxic effect of different concentrations $(5,10,20$ and $50 \mathrm{mg} / \mathrm{L})$ of the metal on the 
development of the fungus increased efficiency of remediation (Ef.\%). Aspergillus niger; obtained greater EF. remediation (97\%) with respect to Penicillium expansum (95\%) and P. islandicum (94\%), $50 \mathrm{mg} / \mathrm{L}$ of $\mathrm{Cr}(\mathrm{VI})$-contaminated waste. It was determined that the presence of $\mathrm{Cr}(\mathrm{VI})$ and not its concentration stimulated early maturation (48 h) of conidia (white on black) from $A$. niger, unless you observe alterations in the mycelium as compared to the control $(72 \mathrm{~h})$, developed in the pulp without the metal. In conclusion, $A$. niger was stronger and presented high Ef. remediation of $\mathrm{Cr}(\mathrm{VI})$ waste, this process is an alternative to physico-chemical technologies, due to the micro-organisms be removed selectively different ions from contaminated areas.

\section{INTRODUCCION}

El cromo, es un metal pesado, que se aplica en procesos industriales como el curtido de cueros, electrónica, fabricación de colorantes, plaguicidas y fotograbados, por lo cual es el contaminante común de los desechos y efluentes industriales en forma de $\mathrm{Cr}(\mathrm{VI})$ (1). Las principales vías de intoxicación de éste metal, son por ingestión, inhalación y contacto con la piel, produciendo irritación del conducto gastrointestinal, perforaciones de la mucosa respiratoria y úlceras dérmicas. Por el contrario, otra especie del cromo, el $\mathrm{Cr}$ (III), es relativamente inocuo debido a la incapacidad para atravesar las membranas biológicas. Nutricionalmente, es un componente esencial en la dieta de todos los organismos, porque interviene en el metabolismo de la glucosa, proteínas y lípidos (2). La Agencia Internacional para la Investigación del Cáncer (International Agency for Research on Cancer IARC), clasificó al Cr(VI) en el Grupo I, que corresponde a sustancias comprobadamente cancerígenas, mientras que al $\mathrm{Cr}$ (III) se incluyó en el Grupo II, como no cancerígenos (3). Los metales pesados no son ni química ni biológicamente degradables, por lo cual, una vez emitidos, pueden permanecer en el ambiente y ser adsorbido 0 asimilado por los vegetales u otros organismos, que al ser ingeridos por los animales, se trasmiten a la cadena alimentaria.

Las industrias reducen el contenido de metales pesados, de los efluentes o residuos, por tratamientos físicos y químicos como precipitación, ósmosis inversa, adsorción, etc. Estos métodos son efectivos pero tienen la desventaja de tener elevados costos, debido al precio de los productos químicos (4). Una alternativa a estos tratamientos, es la biorremediación, tecnología emergente que utiliza organismos vivos (plantas, algas, hongos y bacterias) que adsorben, degradan 0 transforman Ios contaminantes para eliminarlos, inactivarlos 0 atenuar su efecto (5). Los microorganismos, desempeñan un papel importante en la captación de metales tóxicos del medio ambiente, mediante mecanismos de biotransformación, bioacumulación, biosorción (bioadsorción) e inmovilización. Estos mecanismos se aplican en los ciclos biogeoquímicos en los cuales aparecen variantes microbianas capaces de tolerar los efectos nocivos de los metales, de allí surgen los microorganismos resistentes, caracterizados por poseer mecanismos de detoxificación codificados genéticamente e inducibles por el metal, y los tolerantes desarrollan indiferentes a la presencia del metal(6). Los microorganismos más estudiados en procesos de remediación son las bacterias debido que reducen el $\mathrm{Cr}(\mathrm{VI})$ tóxico a $\mathrm{Cr}(\mathrm{III})$ menos tóxico $(7,8)$ y menos estudiados son los hongos filamentosos $(9,10)$. De acuerdo a la bibliografía, las células fúngicas interaccionan con el $\mathrm{Cr}(\mathrm{VI})$ y $\mathrm{Cr}(\mathrm{III})$ a diferentes niveles (Figura 1), desde la pared celular, el periplasma y la membrana plasmática, hasta el citoplasma y las organelas celulares (11). Estos microorganismos necesitan regular los niveles intracelulares de cromo, para mantener un balance entre la incorporación y expulsión del metal. $(6,10,12,13,14)$.

Los metales contenidos en efluentes industriales que son vertidos al suelo o ríos pueden ser adsorbidos por residuos sólidos depositados en el suelo o ser disueltos en el agua. Debido a esto, los microorganismos empleados en procesos de remediación deben tener la capacidad de colonizar y utilizar los residuos sólidos como fuentes de carbono (15). El objetivo del trabajo fue estudiar la biorremediación de un residuo como pulpa de limón contaminada con $\mathrm{Cr}(\mathrm{VI})$ y el efecto del metal sobre el crecimiento fúngico. 


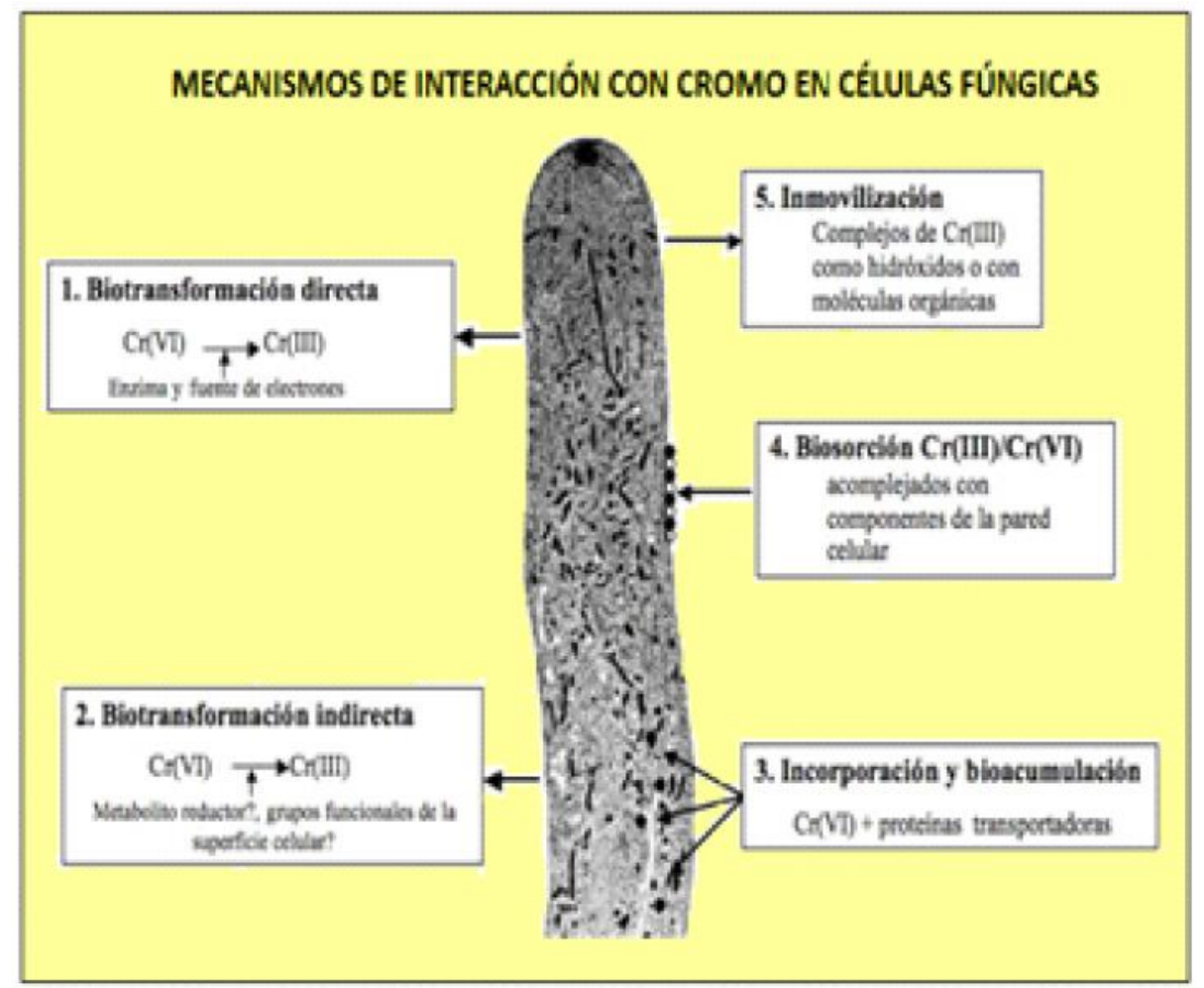

Figura 1: Mecanismos de interacción de la célula fúngica con $\operatorname{Cr}(\mathrm{Vl})$ y $\operatorname{Cr}(\mathrm{III})(14)$

\section{MATERIALES Y MÉTODOS}

I-Evaluación de la adsorción de $\operatorname{Cr}(\mathrm{VI})$ en el residuo cítrico: Para este ensayo se siguieron los siguientes pasos:

1-Adsorbente: Se utilizó pulpa de limón residual (residuo) de una Citrícola (Tucumán, Argentina) que se secó hasta un $80 \%$ de humedad, para la adsorción de $\mathrm{Cr}(\mathrm{VI})$ de una solución acuosa, a fin de obtener un residuo sólido contaminado con el metal para los ensayos de biorremediación.

2-Preparación de las soluciones de $\operatorname{Cr}(\mathrm{VI})$ : $\mathrm{Se}$ preparó una solución patrón de $\mathrm{Cr}(\mathrm{VI})$ de $500 \mathrm{mg} / \mathrm{L}$ a $\mathrm{pH} 2,0\left(\mathrm{H}_{2} \mathrm{SO}_{4}\right.$ al $\left.20 \%, \mathrm{v} / \mathrm{v}\right)$. De ésta solución se preparó una concentración de $\mathrm{Cr}(\mathrm{VI})$ de $50 \mathrm{mg} / \mathrm{L}$ para un volumen final de $50 \mathrm{ml}$. En cada solución se verificó la concentración inicial del metal y el pH inicial.

3-Proceso de adsorción de $\mathrm{Cr}(\mathrm{VI})$ por pulpa de limón: Se pesó $20 \mathrm{~g}$ de pulpa y se colocó (por duplicado) en frascos de Erlenmeyer con $50 \mathrm{ml}$ de la solución de $\mathrm{Cr}(\mathrm{VI})$ de $50 \mathrm{mg} / \mathrm{L}$ (a pH 2,0). Para la adsorción del metal, los Erlenmeyer se colocaron en un agitador rotatorio a $30^{\circ} \mathrm{C}$ y $100 \mathrm{rpm}$ durante $1 \mathrm{~h}$. Posteriormente, la pulpa (adsorbente) se separó de la solución por filtración con papel de filtro. En el filtrado obtenido se determinó $\mathrm{Cr}(\mathrm{VI})$ residual $(\mathrm{Cr}(\mathrm{VI}) \mathrm{r}$ ) y el papel de filtro usado, fue disgregado y colocado en tubo con $5 \mathrm{ml}$ de agua destilada (por triplicado) para determinar si el metal fue retenido por el papel. La cantidad de $\mathrm{Cr}(\mathrm{VI})$ adsorbido en la pulpa, fue determinado por la ecuación: $\operatorname{Cr}(\mathrm{VI}) \mathrm{p}=\mathrm{Cr}(\mathrm{VI}) \mathrm{i}-$ $\mathrm{Cr}(\mathrm{VI}) r$. Donde: $\mathrm{Cr}(\mathrm{VI}) p$, es concentración del metal retenido por la pulpa; $\mathrm{Cr}(\mathrm{VI}) \mathrm{i}$, concentración inicial de la solución preparada y $\mathrm{Cr}(\mathrm{VI})$ r, concentración del metal residual en el filtrado, obtenido después de extraer la pulpa (17).

\section{II- Biorremediación de la pulpa contaminada con Cr(VI) por hongos filamentosos:}

Para este ensayo se realizaron los siguientes pasos

1-Microorganismos: Se utilizaron Aspergillus niger (16); Penicillium expansum y Penicillium islandicum, del cepario de la Cátedra de Micología.

2-Mantenimiento de las cepas: Los microorganismos se mantuvieron activos por repiques sucesivos en medio Czapek modificado, que contiene, 
en g/L: glucosa, 10; $\mathrm{NaNO}_{3}, 3 ; \mathrm{KH}_{2} \mathrm{PO}_{4}, 1 ; \mathrm{MgSO}_{4} .7 \mathrm{H}_{2} \mathrm{O}$, 0,5; KCl, 0,5; agar, 15 y se llevó a pH 4,5 $\mathrm{Con}_{2} \mathrm{SO}_{4}(20$ $\%, v / v)$. Las cepas se sembraron en tubos con este medio y se incubaron a $28{ }^{\circ} \mathrm{C}$ durante $96 \mathrm{~h}$, posteriormente se mantuvieron a $4^{\circ} \mathrm{C}$ hasta su utilización.

\section{3- Preparación de la suspensión de conidios: La} suspensión se preparó para cada hongo en estudio, adicionando conidios a $50 \mathrm{ml}$ de solución fisiológica y $0,05 \mathrm{ml}$ de tween 80 , hasta alcanzar una densidad óptica de 0,250 , que equivale a $2 \times 10^{6}$ conidios $/ \mathrm{ml}$, para una longitud de onda de $560 \mathrm{~nm}$.

4- Ensayos de biorremediación: Para cada hongo se usaron distintas cajas de Petri con $20 \mathrm{~g}$ de pulpa de limón contaminada con $\mathrm{Cr}(\mathrm{VI})(50 \mathrm{mg} / \mathrm{L})$ suplementada con urea, 0,006; $\left(\mathrm{NH}_{4}\right)_{2} \mathrm{SO}_{4}, 0,013$; $\mathrm{KH}_{2} \mathrm{PO}_{4}, 0,003$ y KCl, $0,001 \mathrm{~g} / \mathrm{g}$ de pulpa. Todas las cajas se esterilizaron a $121^{\circ} \mathrm{C}$ por 15 minutos. Posteriormente, se inoculó con $1 \mathrm{ml}$ de suspensión de esporos con $2 \times 10^{6}$ conidios para obtener $10^{5} \mathrm{conidios} / \mathrm{g}$ de adsorbente. Paralelamente, se realizaron ensayos control para cada hongo, en cajas de Petri con pulpa sin $\mathrm{Cr}(\mathrm{VI})$, las sales anteriores y el inoculo del microorganismo en estudio. Todos los ensayos se incubaron en estufa a $30^{\circ} \mathrm{C}$ durante $96 \mathrm{~h}$.

Una vez finalizado el proceso de biorremediación, a la pulpa fermentada (sistema pulpa contaminada con el desarrollo del microorganismo) se agregó $15 \mathrm{~mL}$ de agua destilada y se agitó durante 30 minutos para liberar el $\mathrm{Cr}(\mathrm{VI})$ adsorbido que pudo haber quedado en la superficie del sistema pulpa-microorganismo. Para separar la pulpa, se filtró usando membranas filtrantes de 0,45 $\mu \mathrm{m}$, y el filtrado se usó para determinar $\mathrm{Cr}(\mathrm{VI})$ residual. La membrana filtrante se lavó con agua destilada $(1 \mathrm{~mL}$ ) y en las aguas de lavado se midió $\mathrm{Cr}(\mathrm{VI})$ residual para verificar que el metal no fue adsorbido por dicha membrana.

III-Efecto del Cr(VI) sobre las estructuras macroscópicas del hongo seleccionado: Se realizó una cinética de crecimiento del hongo seleccionado por la Ef. de remoción alta en cultivos en superficie, para determinar posibles modificaciones del micelio y elementos de fructificación, debido a la presencia de diferentes concentraciones de $\mathrm{Cr}(\mathrm{VI}): 5 ; 10 ; 20$ y 50 $\mathrm{mg} / \mathrm{L}$. Las cajas de Petri con pulpa contaminada y suplementada con sales fueron inoculadas con $1 \mathrm{~mL}$ de una solución de $2 \times 10^{6}$ conidios para obtener $10^{5}$ conidios/ g de pulpa, a pH 2,5 e incubadas a $30^{\circ} \mathrm{C}$. Paralelamente, se realizaron ensayos control, en las mismas condiciones de cultivo anteriores y sin la adición del metal. Se tomaron muestras cada $24 \mathrm{~h}$ y se observaron en un microscopio (40x), Leica DM 500.

\section{Determinaciones}

$\mathrm{Cr}(\mathrm{VI})$ por el Método de Difenilcarbazida: Se tomó 2,5 mL de filtrado, se ajustó a pH 2,0 con $\mathrm{H}_{2} \mathrm{SO}_{4}$ al 20 $\%$ (v/v), se agregó $0,5 \mathrm{~mL}$ de 1,5 - difenilcarbazida (DFC) $(5 \mathrm{mg} / \mathrm{mL}$ ) y se dejó reposar $10 \mathrm{~min}$. para el desarrollo total de color. Posteriormente, se midió la densidad óptica (D0) de las muestras a una longitud de onda de $540 \mathrm{~nm}$. Los valores se obtuvieron de una curva patrón D0 vs $\mathrm{Cr}(\mathrm{VI})$, (mg/L) (18).

pH en sólidos: Se pesó $5 \mathrm{~g}$ de muestra (pulpa más microorganismo) y se añadió $10 \mathrm{~mL}$ de agua destilada, se agitó por 20 min., se dejó reposar 10 min. y se midió el pH en un equipo marca Microtec (19).

\section{Cálculos matemáticos}

Determinación de q: se define como cantidad de metal retenido por gramo de adsorbente (en peso seco) y se calcula por la siguiente ecuación: $q=(\mathrm{Ci}-\mathrm{Cr}) \times \mathrm{Vt} / \mathrm{Ms}$. Donde $\mathrm{Ci}$, es concentración inicial de $\mathrm{Cr}(\mathrm{VI})$ en la solución; $\mathrm{Cr}$, concentración residual de $\mathrm{Cr}(\mathrm{VI})$ en la solución remediada; Vt, volumen de la solución a remediar (50 $\mathrm{ml}$ expresado en litro) y Ms, masa del bioadsorbente, expresado en gramo de peso seco (p.s.) (17).

Eficiencia de adsorción (Ef) de la pulpa (adsorbente): Es el porcentaje de metal retenido por el adsorbente, y se calcula por: Ef $(\%)=[(\mathrm{Ci}-\mathrm{Cr}) / \mathrm{Ci}] \times 100$. Donde $\mathrm{Ci}$, es concentración inicial de $\mathrm{Cr}(\mathrm{VI})$ en la solución; $\mathrm{Cr}$, concentración residual del metal en la solución remediada.

Eficiencia de remediación $\left(\mathrm{Ef}_{\mathrm{r}}\right)$ : Es el porcentaje de remoción de $\mathrm{Cr}(\mathrm{VI})$ de la pulpa por el microorganismo y se calcula por: $\mathrm{Ef}_{\mathrm{r}}(\%)=[(\mathrm{Ci}-\mathrm{Cr}) / \mathrm{Ci}] \times 100$. Donde $\mathrm{Ci}$, es concentración inicial de $\mathrm{Cr}(\mathrm{VI})$ retenido por la pulpa; $\mathrm{Cr}$, concentración residual de $\mathrm{Cr}(\mathrm{VI})$ en la pulpa fermentada (pulpa más microorganismo) 
Reproducibilidad de los resultados: Todos los experimentos se realizaron por duplicado y en ensayos separados. Los valores son el promedio de dos mediciones. Los mismos se analizaron para obtener la desviación estándar con un MS-Excel y calculados para $\mathrm{F}=95 \%$ (grado de confianza).

\section{RESULTADOS}

I-Evaluación de la adsorción de $\mathrm{Cr}(\mathrm{VI})$ en el residuo cítrico: La eficiencia de adsorción de la pulpa para una concentración de $\mathrm{Cr}(\mathrm{VI})$ de $50 \mathrm{mg} / \mathrm{L}$ fue $98,8 \%$. Durante la adsorción, el adsorbente no modificó el pH $(2,5)$ de la solución, acidez ideal para la solubilidad del metal y la retención por el adsorbente.

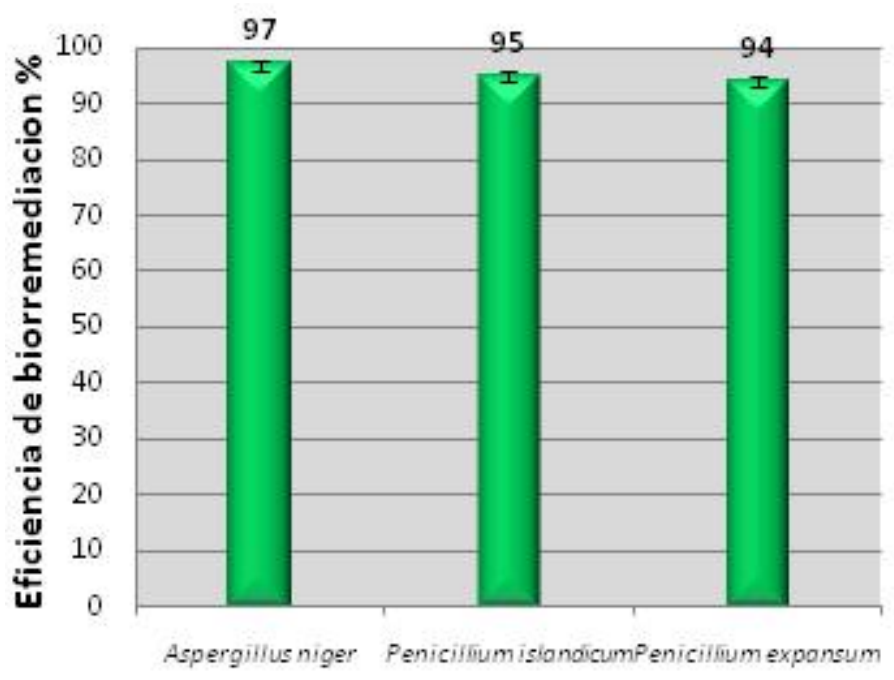

Figura 2: Eficiencia de remediación de $\mathrm{Cr}(\mathrm{VI})[50 \mathrm{mg} /$ L] en la pulpa con diferentes hongos filamentosos. Valores calculados a las $96 \mathrm{~h}$ de incubación.

\section{II- Biorremediación de la pulpa contaminada con} Cr(VI) por hongos filamentosos: Los tres hongos, en estudio, mostraron eficiencias de biorremediación mayor al $90 \%$, siendo Aspergillus niger el que obtuvo la máxima (Figura 2), mientras que con Penicillium islandicum y $P$. expansum, la misma fue 5 y $6 \%$ menor, respectivamente. Con todos los microorganismos, el desarrollo microbiano se evidenció por el aumento del pH inicial de la pulpa de 2,5 a pH 7,0 al final del proceso, debido a la asimilación de la urea y desprendimiento de amoníaco como fue reportado en la bibliografía(20).
III-Efecto del $\operatorname{Cr}(\mathrm{VI})$ sobre las estructuras macroscópicas del hongo seleccionado: El Cr(VI) adsorbido en la pulpa no produjo inhibición del crecimiento de Aspergillus niger, que fue el hongo seleccionado por mayor Ef. de remoción. Todas las concentraciones de $\mathrm{Cr}(\mathrm{VI})$ ensayadas produjeron el ennegrecimiento prematuro de los cultivos (figura 3 ,byc) a las $48 \mathrm{~h}$ de incubación, respecto al cultivo control (figura 3a) a ese tiempo. Este oscurecimiento se debe que el metal creó un ambiente de estrés que estimuló la maduración de los conidios de claros a oscuros como se observa en la figura $4 \mathrm{~b}$, respecto al control (fig. 4 a). Análisis microscópicos de las hifas y cuerpos de fructificación de $A$. niger no mostraron alteración o engrosamiento de hifas, lo cual indica que el $\mathrm{Cr}(\mathrm{VI})$ a $50 \mathrm{mg} / \mathrm{L}$ no tuvo un efecto negativo sobre el desarrollo del hongo.

\section{DISCUSION}

La pulpa de limón residual de la industria citrícola, presentó un valor de $q=1,73 \mathrm{mg} / \mathrm{g}$ (peso seco), similar a los reportados con biomasa viva de Saccharomyces cerevisiae $(2,0 \mathrm{mg} / \mathrm{g})(21)$ y $1,2 \mathrm{mg} / \mathrm{g}$ para biomasa inactiva de Aspergillus niger A2 (22), pero difiere del valor encontrado $(q=4,3 \mathrm{mg} / \mathrm{g})$ para Rhizopus $s p$. (23). La pulpa presentó alta Ef. de adsorción de $\mathrm{Cr}(\mathrm{VI})$ y no alteró el pH de la solución a remediar $(\mathrm{pH} 2,5)$. Esto es importante porque a ese $\mathrm{pH}$, la superficie del adsorbente adquiere mayor cantidad de protones, que inducen a una fuerte atracción por los iones $\mathrm{Cr}(\mathrm{VI})$ de la solución, cargados negativamente, por lo cual la adsorción incrementa. Esto muestra que la pulpa al ser vertida a los suelos puede inmovilizar al metal pesado reteniendo la contaminación en el medio ambiente, resultado que coincide con otras bibliografías $(24,25)$. La remediación de estos residuos sólidos contaminados es factible con hongos filamentosos, ya que poseen estrategias para la colonización de los residuos, distintas de las levaduras y bacterias. Esto se debe que los hongos tienen gran variedad de enzimas que hidrolizan celulosa, almidón, pectina, hemicelulosa, etc., de residuos agroindustriales, y cubren la superficie de los sólidos por extensiones y ramificaciones de hifas que componen el micelio (26). 
Mientras que las levaduras y bacterias, se desarrollan mejor en cultivos líquidos $(21,27)$. Los tres hongos ensayados presentaron Ef. de remediación mayores a $90 \%$, lo cual indica que en sistemas de cultivo en superficie, la mayor concentración de $\mathrm{Cr}(\mathrm{VI})$ empleada
(50 mg/L), no produjo inhibición significativa $(\tilde{n}>0,05)$ del crecimiento celular. Pero se evidenció que el $\mathrm{Cr}(\mathrm{VI})$ produjo un medio ambiente extremo que influyó sobre la maduración de los elementos de reproducción y diseminación de A. niger.
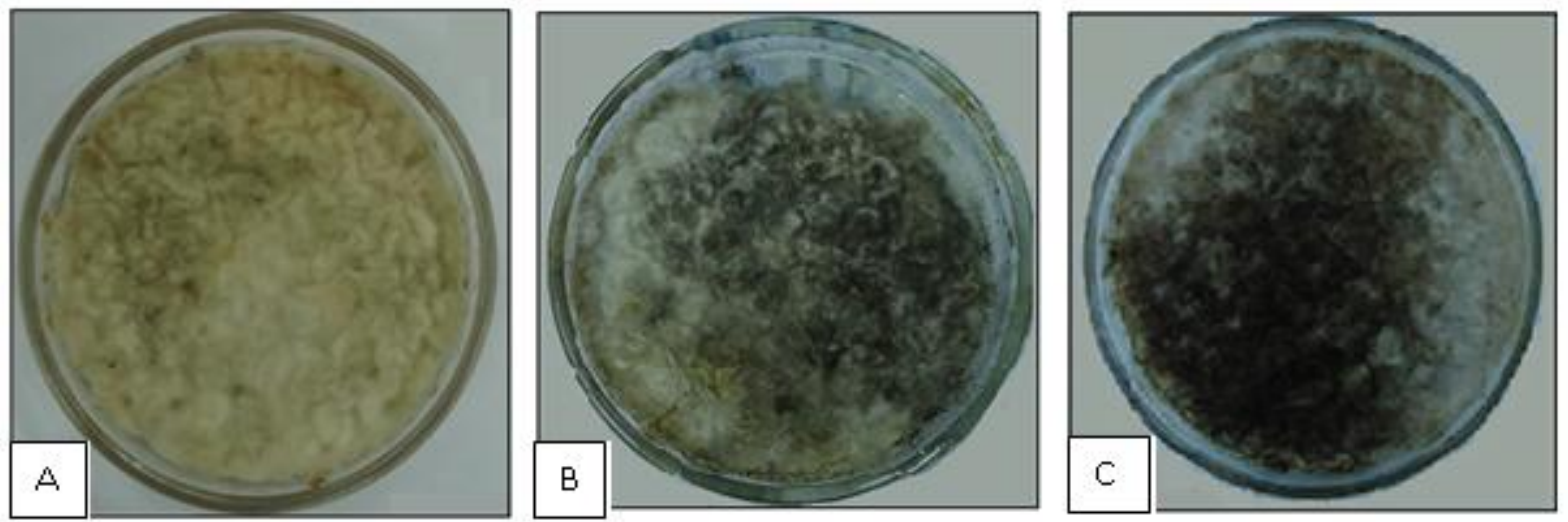

Figura 3: Desarrollo de Aspergillus niger en pulpa: A) sin adición de $\operatorname{Cr}(\mathrm{VI})$; B) con $5 \mathrm{mg} / \mathrm{L}$ y C) con $50 \mathrm{mg} / \mathrm{L}$ del metal. Fotografías tomadas a las $48 \mathrm{~h}$ de incubación.
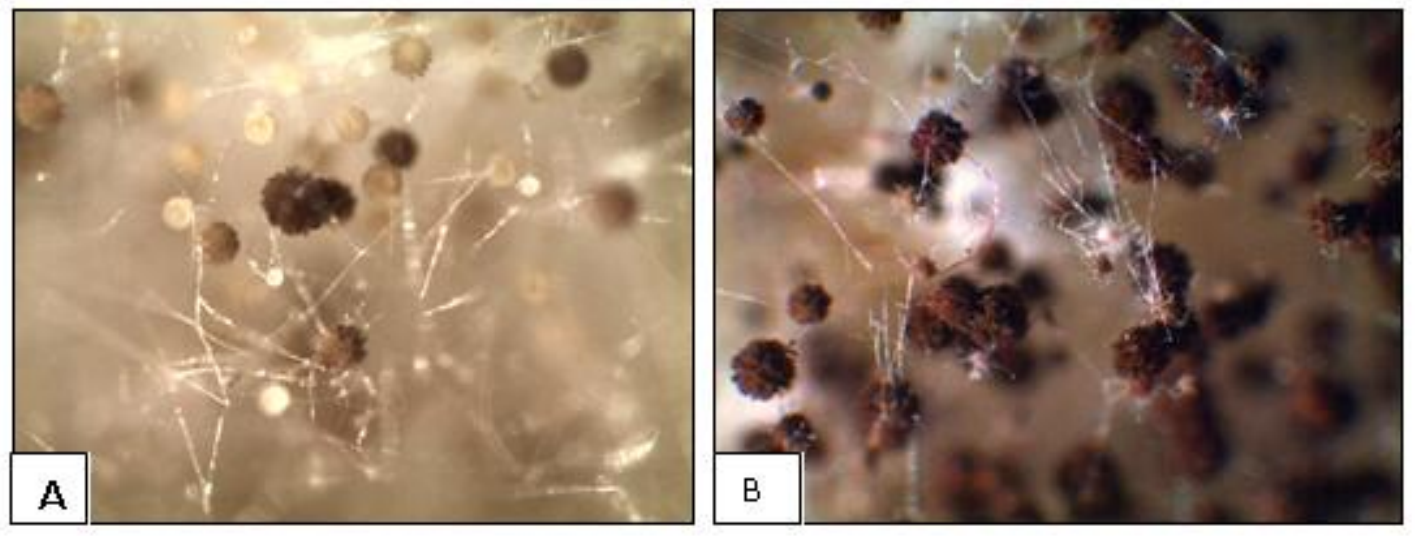

Figura 4: Cabezas aspergilares e hifas de Aspergillus niger desarrollada en, A: Control, hongo crecido en pulpa sin $\mathrm{Cr}(\mathrm{VI})$. y B: pulpa con $\mathrm{Cr}(\mathrm{Vl})[50 \mathrm{mg} / \mathrm{L}]$. Fotografía de $48 \mathrm{~h}$ de incubación en cultivos en superficie (40x microscopio Leitz).

\section{CONCLUSION}

Los resultados de remediación de residuos contaminadas con $\mathrm{Cr}(\mathrm{VI})$ mostraron que, Aspergillus niger fue más resistente a la acción del $\operatorname{Cr}(\mathrm{VI})$ y presentó alta eficiencia de remoción de $\mathrm{Cr}(\mathrm{Vl})$ a 50 $\mathrm{mg} / \mathrm{L}$ de residuos $(97 \%)$ y puede ser empleado en procesos de remediación. Los procesos biotecnológicos aplicados a la purificación de residuos contaminados es una alternativa que tiene grandes ventajas respecto a las tecnologías físico-químicas, debido que las biomasas fúngicas son naturales, se pueden obtener en grandes cantidades a bajo costo, y pueden remover selectivamente diferentes metales de zonas contaminadas.

\section{AGRADECIMIENTOS}

Se agradece al la Universidad Nacional de Tucumán $y$ al Centro de investigaciones de la UNT. 


\section{REFERENCIAS}

1. Ramírez Ramírez, R.; Calvo Méndez, C.; Avila Rodríguez, M.; Gutiérrez Corona, J.F. (2000). Chromate resistance and reduction in a yeast strain isolated from industrial waste discharges. In: Environmental Engineering and Health Sciences. Section 4: Environmental Engineering Application. Water Res. Publ. pp.437-445.

2. Alvarado Gómez, A., Blanco Saénz, R., Mora Moreli, E. (2002). El cromo como elemento esencial en los humanos. Rev. Costarricense de Cs. Med. 23: 55-68.

3. EPA/IRIS (2010). U.S. Environmental Protection Agency. Toxicological Review of Hexavalent Chromium. External Review Draft. Washington.

4. Wood, T.K. (2008). Molecular approaches in bioremediation. Cur. Opinion Biotech. 19: 572-578.

5. Gadd, G.M. (2000). Bioremedial potential of microbial mechanisms of metal mobilization and inmobilization. Curr. Opinion Biotechnol. 11:271-279.

6. Fukuda, T.; Ishino, Y.; Ogawa, A.; Tsutsumi, K.; Morita, H. (2008). Chromium (VI) reduction from contaminated soils by Aspergillus sp. N2 and Penicillium sp. N3 isolated from chromium deposits. J. Gen. Appl. Microbiol. 54: 295-303.

7. Mabbett, A.; Ping, Y.; Peter, J; Farr, G.; Macaskie, L. (2004). Reduction of $\mathrm{Cr}(\mathrm{VI})$ by palladized of Desulfuvibrio desulfuricans ATCC 29577. Biotechnol. Bioeng. 87: 104-109.

8. Otiniano, M.; Tuesta, L.; Robles, H.; Luján, M.; Chávez, M. (2005). Biorremediación de $\mathrm{Cr}(\mathrm{VI})$ de aguas residuales de curtiembres por Pseudomonas sp. y su efecto sobre el ciclo celular de Allium cepa. Rev. Méd. Vallejiana. 4: 32- 42.

9. Acevedo Aguilar, F.J.; Espino Saldaña, A.E.; León Rodríguez, I.L.; Ávila Rodríguez, M.; Wrobel, K.; Wrobel, K.; Lappe, P.; Ulloa, M.; Gutiérrez Corona, J.F. (2006). Hexavalent chromium removal in vitro and from industrial wastes; using chromate-resistant strains of filamentous fungi indigenous to contaminated wastes. Canadian J. Microbiol. 52:809-815.

10. Congeevaram, S.; Dhanarani, S.; Park, J.; Dexilin, M.; Thamaraiselvi, K. (2007). Biosorption of chromium and nickel by heavy metal resistant fungal and bacterial isolates. J. Hazardous Mat.146:270-277.

11. Gutiérrez Corona, J.F.; Espino Saldaña, A.E; Coreño Alonso, A.; Acevedo Aguilar, F.J.; Reyna López, G.E.; Fernández, F.J.; Tomasini, A.; Wrobel, K.; Wrobel, K. (2010). Mecanismos de interacción con cromo y aplicaciones biotecnológicas en hongos. Rev. Lat. Biotecnol. Amb. Algal 1:47-63.

12. Cervantes, C.; Campos García, J.; Devars, S.; Gutiérrez Corona, F.; Loza Tavera, H.; Torres Guzmán, J.C.; Moreno Sánchez, R. (2001). Interactions of chromium with microorganisms and plants. FEMS Microbiol. Rev. 25:335-347.

13. Mala, S.M.J.; Unni, N.B.; Puvanakrishnan, R. (2006). Bioaccumulation and biosorption of chromium by Aspergillus niger MTCC 2594. J. Gen. Appl. Microbiol. 52:179-186.

14. Coreño Alonso, A. (2009). Caracterización del sistema de reducción de $\mathrm{Cr}$ (VI) de la cepa Ed8 de Aspergillus niger resistente a cromato. Tesis de doctorado (Biología), Posgrado en Biología Experimental, Universidad de Guanajuato, Guanajuato.

15. Maldonado, M.C. (2005). Fermentación en sustrato sólido. Navarro, A.; Maldonado, M. C.; Rubio, M. C. (Eds.) En: Biotecnología Microbiana. Universidad Nacional de Tucumán (Edt.). pp:49-65.

16. Rubio, MC and Maldonado, MC (1995). Purification and characterization of invertase from Aspergillus niger. Curr. Microbiol. 31: 80-83.

17. Barrionuevo, M., Daniel, M., Garavaglia, L., Méndez, M., Sosa, G., Candal, R., Cerdeira, S., Ceretti, H., Ramírez, S., Reciulchi, E., Zalts, A., Vullo, 
D. (2009). Tratamiento biológico de efluentes industriales con metales: factores a tener en cuenta para un diseño eficiente. Rev. Qca. Viva. 2: 106-123.

18. Greenberg, A. E.; Clesceri, L., Eaton, A. (1992). Standard methods for the examination of water and wastewater: American Public Health Association. Washington, D.C. 18:358-360.

19. Calderón, F., Pavlova, M. (1999). Metodologías para análisis químico de suelos. http:// www.drcalderonlabs.com.

20. Suárez, M.S, Rubio, M.C. (2009). Enriquecimiento de la pulpa de limón con proteína microbiana para alimento animal. Tesis de Licenciatura en Biotecnología. Facultad de Bioquímica, Química y Farmacia. Universidad Nacional de Tucumán. Argentina.

21. Ferraz, A.; Tavares, T.; Teixeira, J.A. (2004). Cr (III) removal and recovery from Saccharomyces cerevisiae. Chem. Eng. J. 105:11-20.

22. Zafar, S., Aquil, F., Ahmad, I. (2007). Metal tolerance and biosorption potential of filamentous fungi from metal contaminated agricultural soil. Biores. Technol. 98: 2557-2561.
23. Filipovic Kovacevic, Z.; Sipos, L.; Briski, F. (2000). Biosorption of chromium, copper, nickel and zinc ions onto fungal pellets of Aspergillus niger 405 from aqueous solutions. Food Technol. Biotechnol. 38:211216.

24. Goyal, N.; Jain, S.C. y Banerjee, U.C. (2003). Comparative studies on the microbial adsorption of heavy metals. Adv. Env. Res. 7:311-319.

25. Suárez, M.S., Rubio, M.C. (2011). Adsorción de iones $\mathrm{Cr}(\mathrm{VI})$ con residuos de la industria citrícola y su recuperación como producto enriquecido con proteína microbiana. Proyecto beca de iniciación en la investigación. Consejo de Investigación de la Universidad Nacional de Tucumán. Argentina.

26. Sarkar, S., Satheshkumar, A., Premjumar, R. (2013). Hexavalent chromium removal by live mycelium of Trichoderma harzianum strain. Mol. Soil Biol. 4: 1-6.

27. Srinath, T.; Verma, T.; Ramteke, P.; Garg, S. (2002). Chromium (VI) biosorption and bioaccumulation by chromate resistant bacteria. Chemosph. 48:427-433. 